\title{
Use and Control of Artificial Intelligence in Patients Across the Medical Workflow: Single-Center Questionnaire Study of Patient Perspectives
}

Simon Lennartz, MD; Thomas Dratsch, DPhil, MD; David Zopfs, MD; Thorsten Persigehl, MD; David Maintz, MD; Nils Große Hokamp, MD; Daniel Pinto dos Santos, MD

Institute for Diagnostic and Interventional Radiology, Faculty of Medicine and University Hospital Cologne, University of Cologne, Cologne, Germany

\section{Corresponding Author:}

Daniel Pinto dos Santos, MD

Institute for Diagnostic and Interventional Radiology

Faculty of Medicine and University Hospital Cologne

University of Cologne

Kerpener Straße 62

Cologne, 50937

Germany

Phone: 4922147896063

Fax: 4922147882384

Email: daniel.pinto-dos-santos@uk-koeln.de

\section{Abstract}

Background: Artificial intelligence (AI) is gaining increasing importance in many medical specialties, yet data on patients' opinions on the use of $\mathrm{AI}$ in medicine are scarce.

Objective: This study aimed to investigate patients' opinions on the use of AI in different aspects of the medical workflow and the level of control and supervision under which they would deem the application of AI in medicine acceptable.

Methods: Patients scheduled for computed tomography or magnetic resonance imaging voluntarily participated in an anonymized questionnaire between February 10, 2020, and May 24, 2020. Patient information, confidence in physicians vs AI in different clinical tasks, opinions on the control of AI, preference in cases of disagreement between AI and physicians, and acceptance of the use of AI for diagnosing and treating diseases of different severity were recorded.

Results: In total, 229 patients participated. Patients favored physicians over AI for all clinical tasks except for treatment planning based on current scientific evidence. In case of disagreement between physicians and AI regarding diagnosis and treatment planning, most patients preferred the physician's opinion to AI (96.2\% [153/159] vs 3.8\% [6/159] and 94.8\% [146/154] vs 5.2\% [8/154], respectively; $P=.001$ ). AI supervised by a physician was considered more acceptable than AI without physician supervision at diagnosis (confidence rating 3.90 [SD 1.20] vs 1.64 [SD 1.03], respectively; $P=.001$ ) and therapy (3.77 [SD 1.18] vs 1.57 [SD $0.96]$, respectively; $P=.001)$.

Conclusions: Patients favored physicians over AI in most clinical tasks and strongly preferred an application of AI with physician supervision. However, patients acknowledged that AI could help physicians integrate the most recent scientific evidence into medical care. Application of AI in medicine should be disclosed and controlled to protect patient interests and meet ethical standards.

(J Med Internet Res 2021;23(2):e24221) doi: 10.2196/24221

\section{KEYWORDS}

artificial intelligence; clinical implementation; questionnaire; survey

\section{Introduction}

The incremental use of artificial intelligence (AI) is widely considered as one of the most disruptive developments of the past decades [1]. In medicine, increasing evidence has revealed the promising applications of AI for disease prevention, diagnosis, and treatment [2-4]. Among these, specialties relying on the interpretation of medical imaging data, such as 
dermatology [5], pathology [6], and radiology [7-9], have a particular scientific and economic focus.

While several studies suggested that AI might outperform board-certified physicians at narrow diagnostic tasks [10-13], broad clinical implementation of such technologies has not matched the pace of scientific advancements. Among practical reasons, such as high heterogeneity in clinical data and clinical workflows as well as cost efficiency considerations affecting clinical implementation, other concerns pertain to ethical questions and liability. Consequently, different concepts of AI implementation in existing clinical workflows in a controlled and responsible manner have been discussed to preserve pivotal pillars of accountability [14]. In this regard, one important consensus is that AI should remain as transparent and explainable as possible.

To implement AI in an acceptable manner, it is important to understand perspectives on AI use in clinical routines from among stakeholders, including patients and health care professionals such as students, physicians, and caregivers [15]. In this respect, most studies have reported that medical professionals in specialties most evidently influenced by AI agree with its implementation and consider it a tool complementing the armamentarium they regularly work with. Despite dire early predictions_-for example, radiologists being potentially replaced by AI [16]-recent studies have demonstrated a more gradual adaptation of AI solutions that currently augment human capabilities rather than replacing them [17].

Regarding patients' perspectives on the use of AI in medicine, current studies are primarily focused on particular subspecialties or individual diagnostic procedures [18-20]. Although most of these studies indicate that patients generally accept the implementation of AI in medicine, a recent study investigating patients' perspectives on implementing specific AI devices revealed controversial opinions among numerous patients, particularly regarding the question of human control [21].

Therefore, this study aimed to investigate patients' perspectives on the clinical implementation of AI in a more coherent approach that includes the assessment of key clinical competencies such as physician-patient interaction, diagnosis, and therapy as integral parts of the medical workflow. Further, we investigated opinions for human control of AI and its acceptance depending on different disease severities.

\section{Methods}

\section{Participants}

After our single-center survey study was approved by the institutional review board (approval number 19-1552), patients scheduled for cross-sectional imaging between February 10, 2020, and May 24, 2020, were informed about the possibility to voluntarily complete an anonymous questionnaire on registration. Dedicated boxes for returning the completed questionnaires were placed in the waiting areas. Questionnaires were collected and data were manually transferred to a structured spreadsheet (Excel, Microsoft Corp) at the end of the acquisition period. The response rate was calculated as proposed by the
American Association for Public Opinion Research, using the following formula:

$$
\text { Response rate }=\frac{(\mathrm{C}+\mathrm{P})}{(\mathrm{C}+\mathrm{P})+(R)}
$$

where $\mathrm{C}$ is the number of completed questionnaires; $\mathrm{P}$, the number of partially completed questionnaires; and $\mathrm{R}$, the number of nonresponders not consenting to participate or opting out of the questionnaire study.

\section{Questionnaire}

A senior expert in medical AI, a radiology resident with 4 years of experience and a $\mathrm{PhD}$ in psychology, conducted a literature review on previous surveys on $\mathrm{AI}$ in general and in medicine. As previous surveys were focused on particular subspecialties or limited in their scope, a new survey was generated. Patient interaction, diagnostics, and treatment decisions were identified as key clinical competencies and were therefore implemented as central elements to assess patients' acceptance towards the application of AI in medicine. Because no previous questionnaire comprising all the specific endpoints of our study was available, external validation was omitted. The questionnaire comprised five subsections. The first subsection inquired age, gender, level of education, and a history of a cancer diagnosis. Moreover, prior knowledge of AI had to be indicated. In the second subsection, participants were asked about their confidence in physicians versus AI in different clinical tasks, including the assessment of the medical history of a patient, making of diagnostic and treatment decisions, and addressing of the patients' fears and need for information. The third subsection determined patients' opinions on human control of AI at diagnosis and treatment planning. In the fourth subsection, the respondents were asked to state whose decision should be preferred at diagnosis and treatment planning in case of disagreement between the physician and AI. Finally, in the fifth subsection, participants were asked to indicate their acceptance regarding the application of AI in diagnosing and treating diseases of different severity. In the second, third, and fifth subsections, patients were asked to indicate their agreement based on a Likert scale ranging from 1 ("I strongly disagree") to 5 ("I strongly agree"). In subsection 4, a binary decision between $\mathrm{AI}$ and physician was requested, with the option to choose "I don't know/I don't have an opinion on this."

\section{Sample Size Estimation}

A power analysis was performed for sample size estimation. In order not to disregard smaller, yet important differences in this new research field, our study was sufficiently powered to detect small effects (Cohen $d=0.2$ ). With $\alpha=.05$ and power $=0.80$, the projected sample size needed to detect a small effect (Cohen $d=0.2$ ) for within-group comparisons was $\mathrm{N}=199$ [22]. Therefore, the inclusion of at least 200 participants was deemed necessary.

\section{Statistical Analysis}

Data were analyzed using SPSS (version 25, IBM Corp) as well as R 3.4.0 with RStudio 1.0.136 [23]. Likert scores were compared using two-tailed, paired samples $t$ tests. Chi-squared 
tests were used to compare the proportions of participants. Pearson correlation analysis was used to assess the association between prior knowledge of $\mathrm{AI}$ and confidence on physicians and AI. A $P$ value below .05 was considered statistically significant.

\section{Results}

\section{Participants}

Questionnaire outcomes obtained from 229 patients (99 male, 112 female, 18 of unspecified gender; age 18-82 years)

Table 1. Data obtained from the questionnaire participants ( $\mathrm{N}=229)$. scheduled for computed tomography (CT) or magnetic resonance imaging (MRI) were included. The demographic characteristics, education, prior knowledge of AI and the history of cancer diagnosis of the questionnaire participants are summarized in Table 1. In total, 515 questionnaires were handed out, of which 229 were completed and 19 were incomplete (response rate $48.2 \%)$.

\begin{tabular}{|c|c|}
\hline Characteristic & Participants \\
\hline Age (years), mean (SD) & $51.8(15.4)$ \\
\hline \multicolumn{2}{|l|}{ Gender, $\mathbf{n}(\%)$} \\
\hline Men & $99(43.2)$ \\
\hline Women & $112(48.9)$ \\
\hline Nonbinary & $0(0)$ \\
\hline Not indicated & $18(7.9)$ \\
\hline \multicolumn{2}{|l|}{ Level of education, $n(\%)$} \\
\hline $1\left(\right.$ ISCED $\left.^{\mathrm{a}} 1-2\right)$ & $57(24.9)$ \\
\hline 2 (ISCED 3-5) & $71(31.0)$ \\
\hline 3 (ISCED 6-8) & $84(36.7)$ \\
\hline Not indicated & $17(7.4)$ \\
\hline \multicolumn{2}{|c|}{ Prior knowledge of artificial intelligence (1=completely unfamiliar; $5=$ very familiar), n $(\%)$} \\
\hline 1 & $39(17)$ \\
\hline 2 & $46(20.1)$ \\
\hline 3 & $79(34.5)$ \\
\hline 4 & $28(12.2)$ \\
\hline 5 & $8(3.5)$ \\
\hline Not indicated & $29(12.7)$ \\
\hline \multicolumn{2}{|l|}{ History of oncologic disease, $n(\%)$} \\
\hline Previous cancer diagnosis & $144(62.9)$ \\
\hline No previous cancer diagnosis & $66(28.8)$ \\
\hline Not indicated & $19(8.3)$ \\
\hline
\end{tabular}

${ }^{a}$ ISCED: International Standard Classification of Education. ISCED levels: 1=primary education, $2=$ lower secondary education, $3=$ upper secondary education, $4=$ postsecondary nontertiary education, $5=$ short-cycle tertiary education, $6=$ bachelor or equivalent, $7=$ master or equivalent, $8=$ doctoral or equivalent.

\section{Confidence in the Capabilities of Physicians vs AI}

Patients assigned significantly higher mean scores to the physician rather than to AI for all capabilities included (Table 2 ), except for treatment planning based on the most recent scientific evidence, for which the participants favored AI to physicians (3.96 [SD 0.95] vs 3.71 [SD 0.84]; mean difference $-0.255 ; 95 \% \mathrm{CI}-0.416$ to $-0.094 ; \mathrm{t}_{195}=-3.12 ; P=.002$, Cohen $d=-0.233$ ). Figure 1 summarizes the proportions of ratings assigned to physicians and AI for different clinical tasks. 
Table 2. Comparison of mean ratings regarding confidence in clinical capabilities of physicians and artificial intelligence (AI).

\begin{tabular}{|c|c|c|c|c|c|c|}
\hline Capability & Physician, mean (SD) & $\mathrm{AI}$, mean $(\mathrm{SD})$ & Mean difference $(95 \% \mathrm{CI})$ & $t(d f)$ & $P$ value & Cohen $d$ \\
\hline $\begin{array}{l}\text { Obtaining any relevant informa- } \\
\text { tion from my medical history }\end{array}$ & $3.81(0.85)$ & $3.38(1.04)$ & 0.433 (0.258 to 0.608$)$ & $4.88(177)$ & $<.001$ & 0.407 \\
\hline Making an accurate diagnosis & $3.91(0.76)$ & $3.27(0.96)$ & 0.633 (0.454 to 0.812$)$ & $6.99(168)$ & $<.001$ & 0.617 \\
\hline $\begin{array}{l}\text { Proposing the appropriate } \\
\text { treatment }\end{array}$ & $4.08(0.64)$ & $3.30(0.98)$ & $0.78(0.621$ to 0.94$)$ & 9.67 (172) & $<.001$ & 0.959 \\
\hline $\begin{array}{l}\text { Planning treatment according } \\
\text { to recent state of science }\end{array}$ & $3.71(0.84)$ & $3.96(0.95)$ & $-0.255(-0.416$ to -0.094$)$ & $-3.12(195)$ & .002 & 0.233 \\
\hline $\begin{array}{l}\text { Allocating a sufficient amount } \\
\text { of time for me }\end{array}$ & $3.55(0.99)$ & $3.25(1.30)$ & 0.295 (0.043 to 0.547$)$ & $2.31(172)$ & .02 & 0.208 \\
\hline $\begin{array}{l}\text { Taking away my worries and } \\
\text { addressing my anxieties }\end{array}$ & $4.15(0.86)$ & $2.16(1.08)$ & $1.984(1.79$ to 2.179$)$ & $20.16(192)$ & $<.001$ & 1.653 \\
\hline $\begin{array}{l}\text { Providing all information rele- } \\
\text { vant to my treatment }\end{array}$ & $4.08(0.80)$ & $3.38(1.20)$ & $0.699(0.488$ to 0.911$)$ & $6.52(192)$ & $<.001$ & 0.598 \\
\hline
\end{tabular}

Figure 1. Results of the questionnaire regarding the clinical capabilities of physicians versus artificial intelligence (AI). Percentages refer to the proportion of negative (light orange, orange), neutral (gray), and positive (light blue, blue) responses. Proportions of patients who indicated "Don't know" or left the question blank are indicated on the right. Patients favored physicians to AI for all clinical capabilities except for making a treatment plan based on current clinical knowledge, where they preferred an AI algorithm.

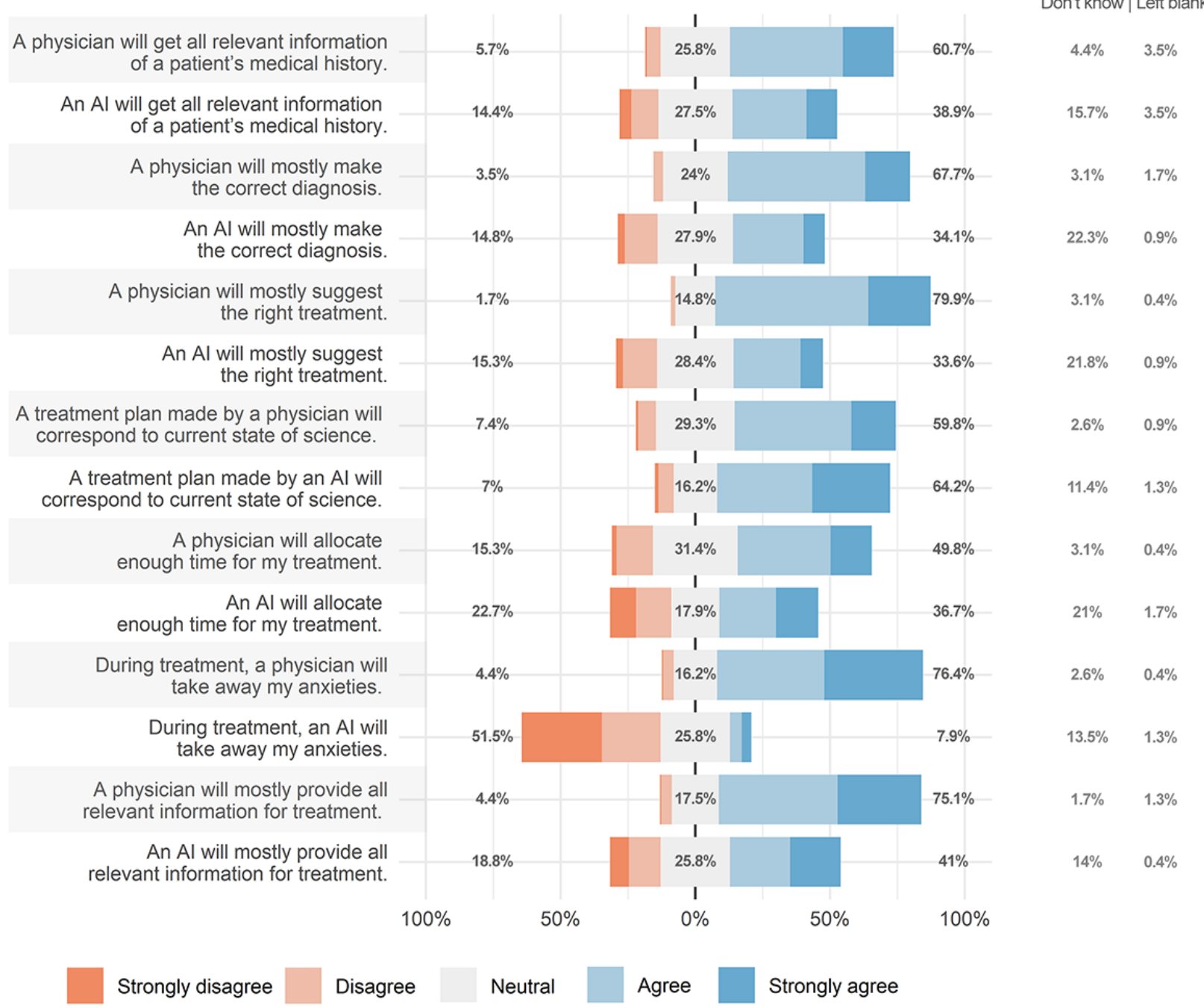


Different Levels of Human Control of AI at Diagnosis and Treatment Planning

During both diagnosis and treatment, patients were significantly more comfortable with the use of AI under the physician's supervision than without such supervision (at diagnosis: 3.90 [SD 1.20] vs 1.64 [SD 1.03]; mean difference 2.26; 95\% CI 2.08 to $2.43 ; \mathrm{t}_{213}=25.19 ; P=.001$; Cohen $d=1.62$; treatment planning: 3.77 [SD 1.18] vs 1.57 [SD 0.96]; mean difference $=2.20 ; 95 \%$ CI 2.03 to $2.38 ; \mathrm{t}_{209}=25.12 ; P=.001$; Cohen $d=1.58)$.

\section{Disagreement Between Physicians and AI}

When asked whose decision should be followed in case of disagreement between the physician and AI regarding the diagnosis, $66.8 \%(153 / 229)$ of patients decided that the diagnosis of the physician should be followed, $2.6 \%(n=6)$ of patients decided that the decision of the AI should be followed, $24.5 \%$ $(n=56)$ of patients responded that they were undecided, and $6.1 \%(n=14)$ of patients did not respond to the question. When analyzing the responses of patients who decided on either AI or a physician, a significantly larger proportion of patients $(153 / 159,96.2 \%)$ decided that the diagnosis of the physician should be considered $\left(\chi^{2}=135.91 ; P=.001\right)$. The same applied to disagreement regarding treatment decisions, for which a similarly large proportion of participants $(146 / 154,94.8 \%)$ decided that the treatment suggested by the physician should be considered $\left(\chi^{2}{ }_{1}=123.66 ; P=.001\right)$.

\section{Application of AI for the Diagnosis and Treatment of Diseases of Different Severity}

There was a significant main effect for disease severity $\left(F_{2,414}=51.75 ; P=.001 ; \eta_{\mathrm{p}}^{2}=0.200\right)$, indicating that the acceptance of AI was lower for more severe diseases than for less severe diseases. Post hoc $t$ tests revealed that the acceptance of AI was significantly lower for diseases of medium severity (3.29 [SD 1.32]) than for those of low severity (3.77 [SD 1.27]; mean difference $=0.48 ; 95 \%$ CI 0.33 to $0.64 ; \mathrm{t}_{211}=6.15 ; P=.001$; Cohen $d=0.426$ ). Additionally, the acceptance of AI was significantly lower for diseases of high severity (2.97 [SD 1.52]) than for diseases of medium severity (3.30 [SD 1.33]; mean difference $=0.33 ; 95 \%$ CI 0.23 to $0.43 ; \mathrm{t}_{207}=6.42 ; P=.001$; Cohen $d=0.498$ ). Figure 2 provides an overview of the proportions of ratings regarding human control of $\mathrm{AI}$, disagreement between $\mathrm{AI}$ and physicians, and acceptance of AI for the diagnosis and treatment of diseases of different severity. 
Figure 2. Results of the questionnaire regarding control of artificial intelligence (AI) (A), disagreement between AI and physicians (B), and the application of AI for diagnosing medical conditions of low, medium, and high severity (C). Percentages refer to the proportion of negative (light orange, orange), neutral (gray), and positive (light blue, blue) responses. Proportions of patients who indicated "Don't know" or left the question blank are indicated on the right.

A I would be fine with an AI making my diagnosis,
if it was checked by an actual physician.
I would be fine with an Al planning my therapy,
if it was checked by an actual physician.
I would be fine with an Al making my diagnosis
without checking by an actual physician.
I would be fine with an Al planning my therapy
without checking by an actual physician. Strongly disagree

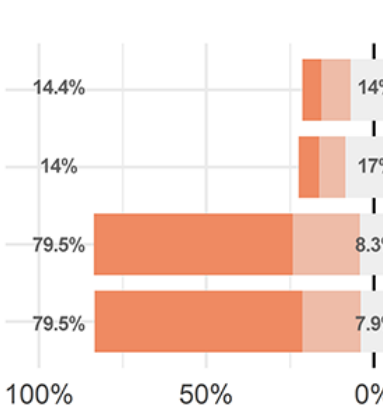
$100 \%$

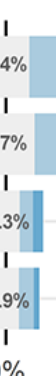

Don't know | Left blank

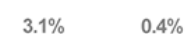

$3.5 \% \quad 0.9 \%$

$\quad 4.8 \% \quad 0.4 \%$

$4.4 \% \quad 2.2 \%$

$\begin{array}{lll}\text { Neutral } & \text { Agree } & \text { Strongly agree }\end{array}$

B

Should my physician and an Al disagree on my diagnosis, whose decision should be preferred? Should my physician and an Al disagree on my treatment, whose decision should be preferred?

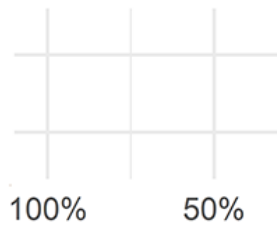

$50 \%$

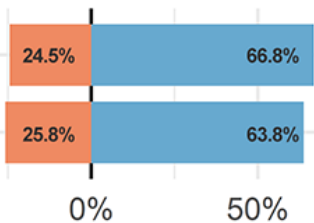

Don't know | Left blank

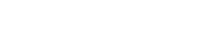

$3.5 \% \quad 7 \%$

$100 \%$

The physician's decision.

The Al's decision.

\section{C}

\section{I would be fine with an Al being
applied for diagnosing less severe medical conditions (e.g. cold, pollen allergy). I would be fine with an Al being applied for diagnosing medium-severe medical conditions (e.g. gall stone, coronary artery disease). \\ I would be fine with an Al being applied for diagnosing severe medical conditions (e.g. trauma from an accident, cancer). \\ Strongly disagree Disagree Neutral \\ Correlation Between Patient-Related Factors and Responses}

Don't know | Left blank

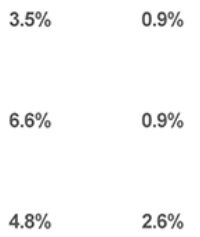

$4.8 \%$ $2.6 \%$
Prior knowledge of AI was by far the most important patient-related factor correlating with certain patients' opinions on AI. As shown in Table 3, prior knowledge of AI was significantly correlated with the acceptance of AI in almost all questionnaire items, indicating that patients assigning a higher rating to their prior knowledge on AI were generally more accepting of the use of AI for various aspects of medical treatment and diagnosis. However, the strength of the correlation was weak overall. 
Table 3. Correlation between prior knowledge of artificial intelligence (AI) and assigned ratings for clinical capabilities of physicians and AI.

\begin{tabular}{|c|c|c|c|c|}
\hline \multirow[t]{2}{*}{ Criteria } & \multicolumn{2}{|c|}{$\begin{array}{l}\text { Correlation between prior knowledge of } \mathrm{AI} \\
\text { and assigned ratings to physicians }\end{array}$} & \multicolumn{2}{|c|}{$\begin{array}{l}\text { Correlation between prior knowledge of } \mathrm{AI} \\
\text { and assigned ratings to AI }\end{array}$} \\
\hline & $r$ & $P$ value & $r$ & $P$ value \\
\hline $\begin{array}{l}\text { Physician/AI would be capable of obtaining any relevant } \\
\text { information from my medical history }\end{array}$ & -0.015 & .83 & .193 & .01 \\
\hline $\begin{array}{l}\text { Physician/AI would be capable of making an accurate di- } \\
\text { agnosis }\end{array}$ & 0.112 & .12 & 0.170 & .03 \\
\hline $\begin{array}{l}\text { Physician/AI would be capable of proposing the appropriate } \\
\text { treatment }\end{array}$ & 0.103 & .16 & 0.213 & .007 \\
\hline $\begin{array}{l}\text { Physician/AI would be capable of planning my treatment } \\
\text { according to recent state of science }\end{array}$ & 0.062 & .39 & 0.295 & $<.001$ \\
\hline $\begin{array}{l}\text { Physician/AI would be capable of allocating a sufficient } \\
\text { amount of time for me }\end{array}$ & 0.012 & .87 & 0.269 & $<.001$ \\
\hline $\begin{array}{l}\text { Physician/AI would be capable of taking away my worries } \\
\text { and addressing my anxieties }\end{array}$ & -0.001 & .99 & 0.310 & $<.001$ \\
\hline $\begin{array}{l}\text { Physician/AI would be capable of providing all information } \\
\text { relevant to my treatment }\end{array}$ & 0.016 & .83 & 0.206 & .007 \\
\hline
\end{tabular}

\section{Discussion}

Over the past few years, research and development in AI has gained considerable momentum [24]. Although AI in medicine is faced with critical challenges regarding its implementation and reimbursement [25], AI technologies will increasingly impact the diagnosis, management, and treatment of diseases in the future.

This study shows that patients would trust physicians over AI in most clinical capabilities except for basing treatment decisions on the most current clinical knowledge, for which AI was considered superior. This is an interesting finding, as it may be increasingly difficult for physicians both in academia and private practice to keep up with the rapidly growing literature in their corresponding subspecialties [26]. Our results indicate that patients seem to be aware of this issue and consider AI superior in incorporating the most recent scientific evidence. It is worth noting that the discrepancy between the acceptance of AI and physicians was largest in the category of "taking away my worries and addressing my anxieties," for which physicians received the highest ratings. While this is, to an extent, an expected result, it clearly underlines the demand for empathetic doctor-patient interactions, concurrent with previous findings [27].

Interestingly, patients favored the capabilities of physicians to AI for diagnosis and treatment decisions, although these two aspects have received extensive media coverage, showing promise in its application in medicine. However, it is important to note that although physicians received higher ratings, a large proportion of patients still had positive views on the clinical capabilities of AI. This "cautious optimism" regarding the usefulness of AI in medicine was reflected by the broad consensus among patients for the use of AI for diagnosing and treating rather mild medical conditions; however, this optimism significantly declined with an increase in disease severity. This is in line with a previous survey outlining that acceptance of AI-based decisions declines when the stakes or risks are higher
[28]. This observation is relevant in view of many commercially available algorithms for diagnosing life-threatening disease conditions such as cerebral hemorrhage, pulmonary embolism, or pneumothorax [29-31].

Because we are in the era of "narrow AI," in which algorithms can fulfil very specific tasks with high accuracy [32], the general conception of AI implementation is to use it as a tool to support clinicians in specific areas. However, it can be expected that with the development of $\mathrm{AI}$ in medicine, some tools might offer broader and more general applications. Different models of implementation of AI in clinical workflows have been conceived, in which the level of autonomy assigned to AI algorithms plays an important role. In this study, most patients reported that AI findings should be double-checked by a physician. In case of a disagreement between physicians and AI, the vast majority of patients (96.2\% [153/159] for disagreement on diagnosis and $94.8 \%$ [146/154] for disagreement on treatment decisions) preferred the physician's to that of AI, concurrent with the results of a previous survey among Chinese cancer patients, in which $88.8 \%$ and $91.3 \%$ of participants reported they would follow the physician's suggestion regarding diagnosis and treatment [27].

The use of AI in medicine without adequate disclosure or explanation to patients can be hazardous [33,34]. Consequently, transparency and explicability are absolutely crucial for AI implementation [14]. Based on these findings on AI control and a significant trend towards lower acceptance rates of AI with increasing disease severity, we conclude that patients should be informed of which tasks involve AI algorithms and whether these applications are supervised by a physician. This is particularly relevant as most AI tools being developed and made available fall under this severe disease category, which explicitly comprised oncologic diseases in our questionnaire.

Our study has limitations that need to be acknowledged. The number of participants we included was rather small, which limits generalization of our results to other populations; for example, a previous study suggested that Asian populations 
may anticipate a more disruptive development of medical AI with the potential replacement of health care professionals [35]. Apart from prior knowledge of AI, we did not observe other significant influences on its acceptance in the medical workflow, which might be attributed to the small sample. However, considering previous reports, we speculate that familiarity with AI technology is indeed the most important factor influencing patients' acceptance of it. Another limitation to consider is that the setting of handing out the questionnaire at registration for cross-sectional imaging, owing to organizational prerequisites, certainly introduced a selection bias towards participants with a history of more severe diseases warranting such radiological examinations. Surveying patients at primary care physician appointments might therefore yield divergent results.

In conclusion, patients had greater confidence in physicians than in AI in most clinical capabilities except for making treatment decisions based on the most recent scientific evidence, where they found AI advantageous. Patients strongly preferred physician-controlled application of AI. In order to safeguard patient interests, disclosure and control of AI application in medicine is crucial.

\section{Acknowledgments}

This study has been partly funded by the Deutsche Forschungsgemeinschaft (DFG, German Research Foundation) - LE 4401/1-1 to SL (project number 426969820), the Else Kröner-Fresenius Stifung (2018_EKMS.34 to NGH) and by the Koeln Fortune Program / Faculty of Medicine, University of Cologne (330/2020).

We thank Melanie Treutlein and Nedim Beste for their support with data collection.

\section{Conflicts of Interest}

None declared.

\section{References}

1. Girasa R. AI as a Disruptive Technology. In: Artif Intell as a Disruptive Technol. Cham, Switzerland: Palgrave Macmillan; 2020:3-21.

2. Graffy PM, Sandfort V, Summers RM, Pickhardt PJ. Automated Liver Fat Quantification at Nonenhanced Abdominal CT for Population-based Steatosis Assessment. Radiology 2019 Nov;293(2):334-342. [doi: 10.1148/radiol.2019190512] [Medline: 31526254]

3. Hinton G. Deep Learning-A Technology With the Potential to Transform Health Care. JAMA 2018 Sep 18;320(11):1101-1102. [doi: 10.1001/jama.2018.11100] [Medline: $\underline{\text { 30178065] }}$

4. Wang C, Zhu X, Hong JC, Zheng D. Artificial Intelligence in Radiotherapy Treatment Planning: Present and Future. Technol Cancer Res Treat 2019 Jan 01;18:1533033819873922 [FREE Full text] [doi: 10.1177/1533033819873922] [Medline: 31495281]

5. Esteva A, Kuprel B, Novoa RA, Ko J, Swetter SM, Blau HM, et al. Dermatologist-level classification of skin cancer with deep neural networks. Nature 2017 Feb 02;542(7639):115-118. [doi: 10.1038/nature21056] [Medline: 28117445]

6. Chang HY, Jung CK, Woo JI, Lee S, Cho J, Kim SW, et al. Artificial Intelligence in Pathology. J Pathol Transl Med 2019 Jan;53(1):1-12 [FREE Full text] [doi: 10.4132/jptm.2018.12.16] [Medline: 30599506]

7. Alexander A, McGill M, Tarasova A, Ferreira C, Zurkiya D. Scanning the Future of Medical Imaging. J Am Coll Radiol 2019 Apr;16(4 Pt A):501-507 [FREE Full text] [doi: 10.1016/j.jacr.2018.09.050] [Medline: $\underline{30522844}$ ]

8. Hosny A, Parmar C, Quackenbush J, Schwartz LH, Aerts HJWL. Artificial intelligence in radiology. Nat Rev Cancer 2018 Aug;18(8):500-510 [FREE Full text] [doi: 10.1038/s41568-018-0016-5] [Medline: 29777175]

9. Choy G, Khalilzadeh O, Michalski M, Do S, Samir AE, Pianykh OS, et al. Current Applications and Future Impact of Machine Learning in Radiology. Radiology 2018 Aug;288(2):318-328 [FREE Full text] [doi: 10.1148/radiol.2018171820] [Medline: 29944078]

10. Rodriguez-Ruiz A, Lång K, Gubern-Merida A, Broeders M, Gennaro G, Clauser P, et al. Stand-Alone Artificial Intelligence for Breast Cancer Detection in Mammography: Comparison With 101 Radiologists. J Natl Cancer Inst 2019 Sep 01;111(9):916-922 [FREE Full text] [doi: 10.1093/jnci/djy222] [Medline: 30834436]

11. Ström P, Kartasalo K, Olsson H, Solorzano L, Delahunt B, Berney DM, et al. Artificial intelligence for diagnosis and grading of prostate cancer in biopsies: a population-based, diagnostic study. Lancet Oncol 2020 Feb;21(2):222-232. [doi: 10.1016/S1470-2045(19)30738-7] [Medline: 31926806]

12. McKinney SM, Sieniek M, Godbole V, Godwin J, Antropova N, Ashrafian H, et al. International evaluation of an AI system for breast cancer screening. Nature 2020 Jan;577(7788):89-94. [doi: 10.1038/s41586-019-1799-6] [Medline: $\underline{31894144]}$

13. Ardila D, Kiraly AP, Bharadwaj S, Choi B, Reicher JJ, Peng L, et al. End-to-end lung cancer screening with three-dimensional deep learning on low-dose chest computed tomography. Nat Med 2019 Jun;25(6):954-961. [doi: 10.1038/s41591-019-0447-x] [Medline: $\underline{31110349]}$

14. Geis JR, Brady AP, Wu CC, Spencer J, Ranschaert E, Jaremko JL, et al. Ethics of Artificial Intelligence in Radiology: Summary of the Joint European and North American Multisociety Statement. J Am Coll Radiol 2019 Nov;16(11):1516-1521. [doi: 10.1016/i.jacr.2019.07.028] [Medline: $\underline{31585696]}$ 
15. Pinto Dos Santos D, Giese D, Brodehl S, Chon SH, Staab W, Kleinert R, et al. Medical students' attitude towards artificial intelligence: a multicentre survey. Eur Radiol 2019 Apr;29(4):1640-1646. [doi: 10.1007/s00330-018-5601-1] [Medline: 29980928]

16. European Society of Radiology (ESR). What the radiologist should know about artificial intelligence - an ESR white paper. Insights Imaging 2019 Apr 04;10(1):44 [FREE Full text] [doi: 10.1186/s13244-019-0738-2] [Medline: 30949865]

17. Arieno A, Chan A, Destounis SV. A Review of the Role of Augmented Intelligence in Breast Imaging: From Automated Breast Density Assessment to Risk Stratification. AJR Am J Roentgenol 2019 Feb;212(2):259-270. [doi: 10.2214/AJR.18.20391] [Medline: 30422711]

18. Nelson CA, Pérez-Chada LM, Creadore A, Li SJ, Lo K, Manjaly P, et al. Patient Perspectives on the Use of Artificial Intelligence for Skin Cancer Screening: A Qualitative Study. JAMA Dermatol 2020 May 01;156(5):501-512. [doi: 10.1001/jamadermatol.2019.5014] [Medline: 32159733]

19. Palmisciano P, Jamjoom AAB, Taylor D, Stoyanov D, Marcus HJ. Attitudes of Patients and Their Relatives Toward Artificial Intelligence in Neurosurgery. World Neurosurg 2020 Jun;138:e627-e633. [doi: 10.1016/j.wneu.2020.03.029] [Medline: 32179185]

20. Haan M, Ongena YP, Hommes S, Kwee TC, Yakar D. A Qualitative Study to Understand Patient Perspective on the Use of Artificial Intelligence in Radiology. J Am Coll Radiol 2019 Oct;16(10):1416-1419. [doi: 10.1016/j.jacr.2018.12.043] [Medline: $\underline{30878311]}$

21. Tran V, Riveros C, Ravaud P. Patients' views of wearable devices and AI in healthcare: findings from the ComPaRe e-cohort. NPJ Digit Med 2019;2:53 [FREE Full text] [doi: 10.1038/s41746-019-0132-y] [Medline: 31304399]

22. Faul F, Erdfelder E, Buchner A, Lang A. Statistical power analyses using G*Power 3.1: tests for correlation and regression analyses. Behav Res Methods 2009 Nov;41(4):1149-1160. [doi: 10.3758/BRM.41.4.1149] [Medline: 19897823]

23. Allaire J. RStudio: integrated development environment for R. RStudio. 2012. URL: http://www.rstudio.com/ [accessed 2021-01-28]

24. Pesapane F, Codari M, Sardanelli F. Artificial intelligence in medical imaging: threat or opportunity? Radiologists again at the forefront of innovation in medicine. Eur Radiol Exp 2018 Oct 24;2(1):35 [FREE Full text] [doi: 10.1186/s41747-018-0061-6] [Medline: 30353365]

25. Banja J. AI Hype and Radiology: A Plea for Realism and Accuracy. Radiology: Artificial Intelligence 2020 Jul 01;2(4):e190223. [doi: 10.1148/ryai.2020190223]

26. Fontelo P, Liu F. A review of recent publication trends from top publishing countries. Syst Rev 2018 Sep 27;7(1):147 [FREE Full text] [doi: 10.1186/s13643-018-0819-1] [Medline: $\underline{\text { 30261915] }}$

27. Yang K, Zeng Z, Peng H, Jiang Y. Attitudes Of Chinese Cancer Patients Toward The Clinical Use Of Artificial Intelligence. Patient Prefer Adherence 2019;13:1867-1875 [FREE Full text] [doi: 10.2147/PPA.S225952] [Medline: 31802856]

28. Smith A. Public Attitudes Toward Computer Algorithms. Pew Research Center Internet \& Technology. 2018. URL: https:/ /www.pewresearch.org/internet/2018/11/16/public-attitudes-toward-computer-algorithms/ [accessed 2021-01-28]

29. Kuo W, H ne C, Mukherjee P, Malik J, Yuh EL. Expert-level detection of acute intracranial hemorrhage on head computed tomography using deep learning. Proc Natl Acad Sci U S A 2019 Nov 05;116(45):22737-22745 [FREE Full text] [doi: 10.1073/pnas.1908021116] [Medline: 31636195]

30. Truong K. Aidoc gets FDA nod for AI pulmonary embolism screening tool. MedCity News. 2019. URL: https://medcitynews. com/2019/05/aidoc-gets-fda-nod-for-ai-pulmonary-embolism-screening-tool/ [accessed 2021-01-28]

31. Shefayim K. Zebra Medical Vision Receives FDA Approval for World's First AI Chest X-ray Triage Product. Zebra Medical Vision. 2019. URL: https://www.prnewswire.com/news-releases/ zebra-medical-vision-receives-fda-approval-for-worlds-first-ai-chest-x-ray-triage-product-300848867.html [accessed 2021-01-28]

32. Davenport T, Kalakota R. The potential for artificial intelligence in healthcare. Future Healthc J 2019 Jun;6(2):94-98 [FREE Full text] [doi: 10.7861/futurehosp.6-2-94] [Medline: $\underline{\text { 31363513] }}$

33. Cohen IG. Informed Consent and Medical Artificial Intelligence: What to Tell the Patient? SSRN Journal 2020;108:1425-1469. [doi: 10.2139/ssrn.3529576]

34. Robins R, Brodwin E. An invisible hand: Patients aren?t being told about the AI systems advising their care Internet. 2020. 2020. URL: https://www.statnews.com/2020/07/15/artificial-intelligence-patient-consent-hospitals/ [accessed 2021-01-27]

35. Gao S, He L, Chen Y, Li D, Lai K. Public Perception of Artificial Intelligence in Medical Care: Content Analysis of Social Media. J Med Internet Res 2020 Jul 13;22(7):e16649 [FREE Full text] [doi: 10.2196/16649] [Medline: 32673231]

\section{Abbreviations}

AI: artificial intelligence

CT: computed tomography

MRI: magnetic resonance imaging 
Edited by G Eysenbach; submitted 10.09.20; peer-reviewed by F Stoehr, $R$ Watson; comments to author 29.09.20; revised version received 01.11.20; accepted 30.11.20; published 17.02.21

Please cite as:

Lennartz S, Dratsch T, Zopfs D, Persigehl T, Maintz D, Große Hokamp N, Pinto dos Santos D

Use and Control of Artificial Intelligence in Patients Across the Medical Workflow: Single-Center Questionnaire Study of Patient

Perspectives

J Med Internet Res 2021;23(2):e24221

URL: http://www.jmir.org/2021/2/e24221/

doi: $\underline{10.2196 / 24221}$

PMID: 33595451

(CSimon Lennartz, Thomas Dratsch, David Zopfs, Thorsten Persigehl, David Maintz, Nils Große Hokamp, Daniel Pinto dos Santos. Originally published in the Journal of Medical Internet Research (http://www.jmir.org), 17.02.2021. This is an open-access article distributed under the terms of the Creative Commons Attribution License (https://creativecommons.org/licenses/by/4.0/), which permits unrestricted use, distribution, and reproduction in any medium, provided the original work, first published in the Journal of Medical Internet Research, is properly cited. The complete bibliographic information, a link to the original publication on http://www.jmir.org/, as well as this copyright and license information must be included. 